\title{
Improvement of precision and sensitivity in refinement of crystal structure factors using zone- axis and Bragg-excited CBED patterns
}

\author{
B. Aryal ${ }^{1}$, D. Morikawa ${ }^{1}$, K. Tsuda ${ }^{2}$, M. Terauchi ${ }^{1}$ \\ ${ }^{1}$ Institute of Multidisciplinary Research for Advanced Materials, Tohoku University, 2-1-1, Katahira, Aoba-ku, \\ Sendai 980-8577, Japan \\ ${ }^{2}$ Frontier Research Institute for Interdisciplinary Sciences, Tohoku University, Aramaki aza Aoba 6-3, Sendai 980-8578, Japan \\ aryal.bikas.p4@dc.tohoku.ac.jp
}

In recent years, convergent-beam electron diffraction (CBED) has been widely used for refining crystal structure parameters and loworder structure factors. It enables nanometer-scale structure analysis with high sensitivity to the distribution of valence electrons. The determination of low-order structure factors with higher precision is essential to precisely determine the chemical bonding state of materials which are closely related to their physical properties. Till date, it is considered that refinement of structure factors using CBED pattern taken at the Bragg-excited condition increases the sensitivity to the corresponding structure factor [1]. However, the origin of precision and relationship between precision and sensitivities of CBED patterns in the refinements of structure factors are still lacking.

Crystal structure analysis method developed by Tsuda and Tanaka [2] has been applied to potassium tantalate $\mathrm{KTaO}_{3}$ (KTO). Isotropic atomic displacement parameters (ADPs) and five low-order structure factors were refined using energy-filtered CBED patterns taken at three zone-axis (ZA) and five Bragg-excited conditions. Precision and sensitivity of ZA and Bragg-excited CBED patterns for refining structure factors are compared. Higher precision in the refinements of structure factors was achieved using the Bragg-excited patterns. The determined values of five low-order structure factors are shown in table 1. Numbers in parenthesis indicate the standard deviations of the last digit. Smaller values of the standard deviations for Bragg-excited condition is indicative of the higher precision. Sensitivity of the Bragg-excited patterns depend on Bragg-condition (dynamical diffraction condition). Higher precision and sensitivity of Bragg-excited CBED pattern has been found only for structure factors of the outer zeroth-order Laue zone (ZOLZ) reflection having larger reciprocal lattice vectors [3]. Smaller correlation coefficients among the refined structure factors in the refinement of Bragg-excited patterns lead to a higher precision. From the point of view of higher precision, Bragg-excited patterns are advantageous over ZA patterns [3]. To achieve higher precision and sensitivities in the refinements of structure factors it would be better to use both ZA and Bragg-excited CBED patterns. The use of large-angle CBED (LACBED) or large-angle rocking beam electron diffraction (LARBED) techniques should be effective for this purpose.

\begin{tabular}{|c|c|c|}
\hline$F_{h k l}(\AA)$ & Zone-axis & Bragg-excited \\
\hline$F_{001}$ & $-6.28(15)$ & $-6.27(2)$ \\
\hline$F_{011}$ & $9.63(14)$ & $9.61(2)$ \\
\hline$F_{\overline{1} 11}$ & $-0.05(10)$ & $0.25(2)$ \\
\hline$F_{002}$ & $11.77(14)$ & $11.50(2)$ \\
\hline$F_{021}$ & $-3.93(13)$ & $-4.05(2)$ \\
\hline
\end{tabular}

Table 1: Five low-order crystal structure factors of $\mathrm{KTaO}_{3}$ determined using $\mathrm{ZA}$ and Bragg-excited CBED patterns.

[1] Ogata, Y., Tsuda, K. \& Tanaka, M. (2008). Acta Cryst. A64, 587.

[2] Tsuda, K. \& Tanaka, M. (1999). Acta Cryst. A55, 939.

[3] Aryal, B., Morikawa, D., Tsuda, K. \& Terauchi, M. (2021). Acta Cryst. A77, 289.

Keywords: CBED; structure factor; precision 